\title{
Single versus multichannel applicator in high-dose-rate vaginal brachytherapy optimized by inverse treatment planning
}

\author{
Yasir A. Bahadur, MD!, Camelia Constantinescu, PhD², Ashraf H. Hassouna, MD ${ }^{3.4}$, Maha M. Eltaher, MD'.4, \\ Noor M. Ghassal, BSc², Nesreen A. Awad, MDl,4 \\ 'Department of Radiology, King Abdulaziz University Hospital, Jeddah, Saudi Arabia, ${ }^{2}$ Department of Bio-Medical Physics, King Faisal \\ Specialist Hospital \& Research Center, Jeddah, Saudi Arabia, ${ }^{3}$ Department of Oncology, King Faisal Specialist Hospital \& Research Center. \\ Jeddah, Saudi Arabia, ${ }^{4}$ Department of Radiation Oncology, National Cancer Institute, Cairo University, Egypt
}

\begin{abstract}
Purpose: To retrospectively compare the potential dosimetric advantages of a multichannel vaginal applicator vs. a single channel one in intracavitary vaginal high-dose-rate (HDR) brachytherapy after hysterectomy, and evaluate the dosimetric advantage of fractional re-planning.

Material and methods: We randomly selected 12 patients with endometrial carcinoma, who received adjuvant vaginal cuff HDR brachytherapy using a multichannel applicator. For each brachytherapy fraction, two inverse treatment plans (for central channel and multichannel loadings) were performed and compared. The advantage of fractional re-planning was also investigated.

Results: Dose-volume-histogram (DVH) analysis showed limited, but statistically significant difference $(p=0.007)$ regarding clinical-target-volume dose coverage between single and multichannel approaches. For the organs-at-risk rectum and bladder, the use of multichannel applicator demonstrated a noticeable dose reduction, when compared to single channel, but statistically significant for rectum only $(p=0.0001)$. For $\mathrm{D}_{2 \mathrm{cc}}$ of rectum, an average fractional dose of $6.1 \pm 0.7 \mathrm{~Gy}$ resulted for single channel vs. $5.1 \pm 0.6 \mathrm{~Gy}$ for multichannel. For $\mathrm{D}_{2 \mathrm{cc}}$ of bladder, an average fractional dose of $5 \pm 0.9$ Gy occurred for single channel vs. $4.9 \pm 0.8 \mathrm{~Gy}$ for multichannel. The dosimetric benefit of fractional re-planning was demonstrated: DVH analysis showed large, but not statistically significant differences between first fraction plan and fractional re-planning, due to large inter-fraction variations for rectum and bladder positioning and filling.

Conclusions: Vaginal HDR brachytherapy using a multichannel vaginal applicator and inverse planning provides dosimetric advantages over single channel cylinder, by reducing the dose to organs at risk without compromising the target volume coverage, but at the expense of an increased vaginal mucosa dose. Due to large inter-fraction dose variations, we recommend individual fraction treatment plan optimization.
\end{abstract}

Key words: endometrial cancer, inverse planning, multichannel applicator, vaginal brachytherapy.

\section{Purpose}

Intracavitary vaginal brachytherapy can be performed either alone or in combination with external beam radiation therapy (EBRT) for patients with endometrial or cervical carcinomas [1,2]. Late radiation reactions after high-dose-rate (HDR) brachytherapy are serious concerns, particularly when the patients are young and sexually active. The risk of late reaction depends on the dose received by the nearby critical organs, as bladder and rectum. Dose optimisation is an old concept in brachytherapy practice, but just recently became a topic for extensive study, due to technological advances in both treatment planning systems and 3D imaging possibilities, as computerized tomography (CT) and magnetic resonance (MR).
Recently, the American Brachytherapy Society (ABS) reported the recommendations for adjuvant vaginal HDR brachytherapy after hysterectomy, establishing dose prescription and optimization guidelines, as well as dose fractionation schedules [3]. Although there is a general consensus to treat the upper third of the vagina as a target for this group of patients because the majority of the treatment failures were reported in this region $[4,5]$, the choice of optimal applicator type is widely varying [3].

The most commonly used applicator for intracavitary vaginal brachytherapy is single channel vaginal cylinder. However, due to its radial symmetry of dose distribution, a single channel applicator offers limited possibilities to optimize the treatment plan according to the patient's
Address for correspondence: Yasir A. Bahadur, MD, Department of Radiology, King Abdulaziz University Hospital, P.O. Box 80215, Jeddah 21589, Saudi Arabia, phone: +966 2 2401000, ext. 17444, mobile: +966 505528528, fax: +966 2 6408047, 凶e-mail: yasirbahadur@hotmail.com
Received: 30.07 .2014

Accepted: 11.11.2014

Published: 30.12.2014 
anatomy. Aiming to improve the capabilities of vaginal brachytherapy, multichannel applicators have been developed. The additional channels at the periphery of the applicator support more conformal dosimetry and amend for the anisotropy generated by a single line source at the vaginal apex [3]. Differential loading of the channels can also potentially reduce the dose to the bladder and rectum, compared with the single channel cylinder.

The purpose of this study is to retrospectively compare, via 3D inverse planning optimization, the potential dosimetric advantages of a multichannel vaginal applicator versus a single channel one, in intracavitary vaginal HDR brachytherapy after hysterectomy. This comparison is made by analyzing dose distributions to target and organs at risk (OARs) volumes.

As the dosimetric and clinical benefits of optimized treatment plans for every fraction are still controversial, we also evaluated the dosimetric advantage of fractional re-planning.

\section{Material and methods}

We are reporting the analysed data of 12 patients with endometrial carcinoma, randomly selected and identified as candidates for vaginal vault brachytherapy, according to our current treatment practice. The patients were treated in the Radiotherapy Unit of King Abdulaziz University Hospital, Jeddah, Saudi Arabia, between January 2012 and November 2013.

The patients had either post-operative EBRT (45 Gy in 25 fractions, one fraction per day, five times per week) to the whole pelvis, using four-fields CT-based planning, followed by HDR brachytherapy (12 Gy in 3 fractions, two fractions per week), or HDR brachytherapy alone (21 Gy in 3 fractions, one fraction per week). However, for the purpose of this study we performed all brachytherapy plans with a prescription dose of 21 Gy in 3 fractions. Brachytherapy was performed with Miami 7-channels vaginal applicator (Varian Medical Systems, Palo Alto, CA, USA), of various diameters $(3$ and $3.5 \mathrm{~cm})$; the applicator has one central and 6 peripheral channels. The peripheral channels produce an asymmetric dose distribution, enabling the treatment plan optimization based on patient anatomy. Patients were requested to evacuate the rectum and bladder just before the applicator insertion. In order to help delineating the bladder and rectum, a $20 \mathrm{cc}$ of Urographine contrast media was injected into the bladder (using Foley's catheter) and $35 \mathrm{cc}$ into the rectum (using a rectal catheter). Computerized tomography images without intravenous contrast were acquired using a Siemens Somatom Emotion CT scanner (Siemens Medical Systems, Erlangen, Germany), with $2 \mathrm{~mm}$ slice intervals from the iliac crest to the distal end of the applicator.

\section{Target and organs at risk delineation}

The clinical target volume (CTV) and the OARs volumes have been delineated on axial CT images for each patient and each brachytherapy fraction prospectively, at the time of each treatment planning, and reviewed retrospectively by one radiation oncologist, for the purpose of this study. The CTV was a $5 \mathrm{~mm}$ expansion of the applicator surface along $5 \mathrm{~cm}$ length measured from the tip of the applicator, and cropped from rectum and bladder. Organ at risk volumes included the rectum and bladder. The outer rectal wall was contoured from the recto-sigmoid junction till $1 \mathrm{~cm}$ above the anal verge, and the outer bladder wall was contoured till the urethra.

Computed tomography image-based planning was performed in Varian Brachyvision planning system, version 10 (Varian Medical Systems, Palo Alto, CA, USA), for a Varian HDR VariSource iX remote afterloader (Varian Medical Systems, Palo Alto, CA, USA). The dose calculation algorithm is based on the TG-43 formalism, as recommended by the American Association of Physicists in Medicine (AAPM) [6]. The inverse planning optimizer identifies the combination of dwell times that best fulfill the dose constraints of target volume and critical organs. Two treatment plans (for both the central channel and multichannel loadings) were performed for each brachytherapy fraction, such that the dose distribution of the two plans could be compared. All plans were optimized by inverse planning, using multicriterial objectives (dose and time), and the following constraints: 1) for CTV coverage: $95 \%$ of the volume to receive 7 Gy and 100\% of the volume to receive 6.65 Gy (95\% of the prescribed dose, as a minimum); 2) for CTV dose homogeneity: source dwell times to not exceed $300 \mathrm{~s}$ for single channel and $100 \mathrm{~s}$ for multichannel applicator; 3 ) for rectum: $2 \mathrm{cc}$ of the volume to receive maximum $4.9 \mathrm{~Gy}$ (70\% of the prescribed dose); 4) for bladder: 2 cc of the volume to receive maximum 5.6 Gy ( $80 \%$ of the prescribed dose).

All dose constraints were set to have the same priority. Dose distributions and dose-volume histograms (DVHs) from these plans were analyzed, and all plans were evaluated and compared using the following indices: the dose covering $100 \%$ of volume $\left(D_{100 \%}\right)$ and $95 \%$ of volume $\left(\mathrm{D}_{95 \%}\right)$ for CTV. As a measure of high dose exposure of vaginal mucosa, the dose received by the most exposed volumes $2 \mathrm{~cm}^{3}\left(\mathrm{D}_{2 \mathrm{cc}}\right)$ of CTV and plan quality indices, such as dose nonuniformity index (DNR, defined as the ratio of CTV receiving a dose equal to or greater than 1.5 times of the prescribed dose to the volume that receives a dose equal to or greater than the prescribed dose; $\left.\mathrm{DNR}=\mathrm{V}_{150 \%} / \mathrm{V}_{100 \%}\right)$ and the overdose index (OI, defined as the ratio of CTV receiving a dose equal to or greater than 2.0 times the prescribed dose to the volume that receives a dose equal to or greater than the prescribed dose; $\left.\mathrm{OI}=\mathrm{V}_{200 \%} / \mathrm{V}_{100 \%}\right)$. Ideally, both quality indices should be equal to zero. In addition, the dose at $5 \mathrm{~mm}$ from the applicator tip was analysed.

For OARs, the doses received by the most exposed volumes of $1 \mathrm{~cm}^{3}\left(D_{1 \mathrm{cc}}\right)$ and $2 \mathrm{~cm}^{3}\left(\mathrm{D}_{2 \mathrm{cc}}\right)$, the volume receiving $70 \%$ of prescription dose $\left(\mathrm{V}_{70 \%}\right)$ for rectum, and the volume receiving $80 \%$ of prescription dose $\left(\mathrm{V}_{80 \%}\right)$ for bladder were considered for comparison. The dosimetric advantage of fractional re-planning was also investigated, using inverse planning optimization and multichannel approach. For each patient, we applied the dwell-time values of the first fraction, corrected for the source decay, to the CT-data set of the subsequent fractions, and compared the dosimetric indices mentioned above to the ones generated by fractional re-planning. 
Table 1. Treatment characteristics

\begin{tabular}{lcc} 
& $\begin{array}{c}\text { No. of } \\
\text { patients } \\
n(\%)\end{array}$ & $\begin{array}{c}\text { No. of } \\
\text { fractions } \\
n(\%)\end{array}$ \\
\hline Multichannel in 3/3 fractions & $3(25 \%)$ & 9 \\
\hline Multichannel in 2/3 fractions & $4(33 \%)$ & 8 \\
\hline Multichannel in 1/3 fractions & $5(42 \%)$ & 5 \\
\hline Total & 12 & 22 \\
\hline Multichannel diameter 3 cm & $10(83 \%)$ & $19(86 \%)$ \\
\hline Multichannel diameter $3.5 \mathrm{~cm}$ & $2(17 \%)$ & $3(14 \%)$
\end{tabular}

The statistical analysis was performed using the Wilcoxon matched pairs test and an in-house software; a $p$ value of $<0.05$ was considered significant.
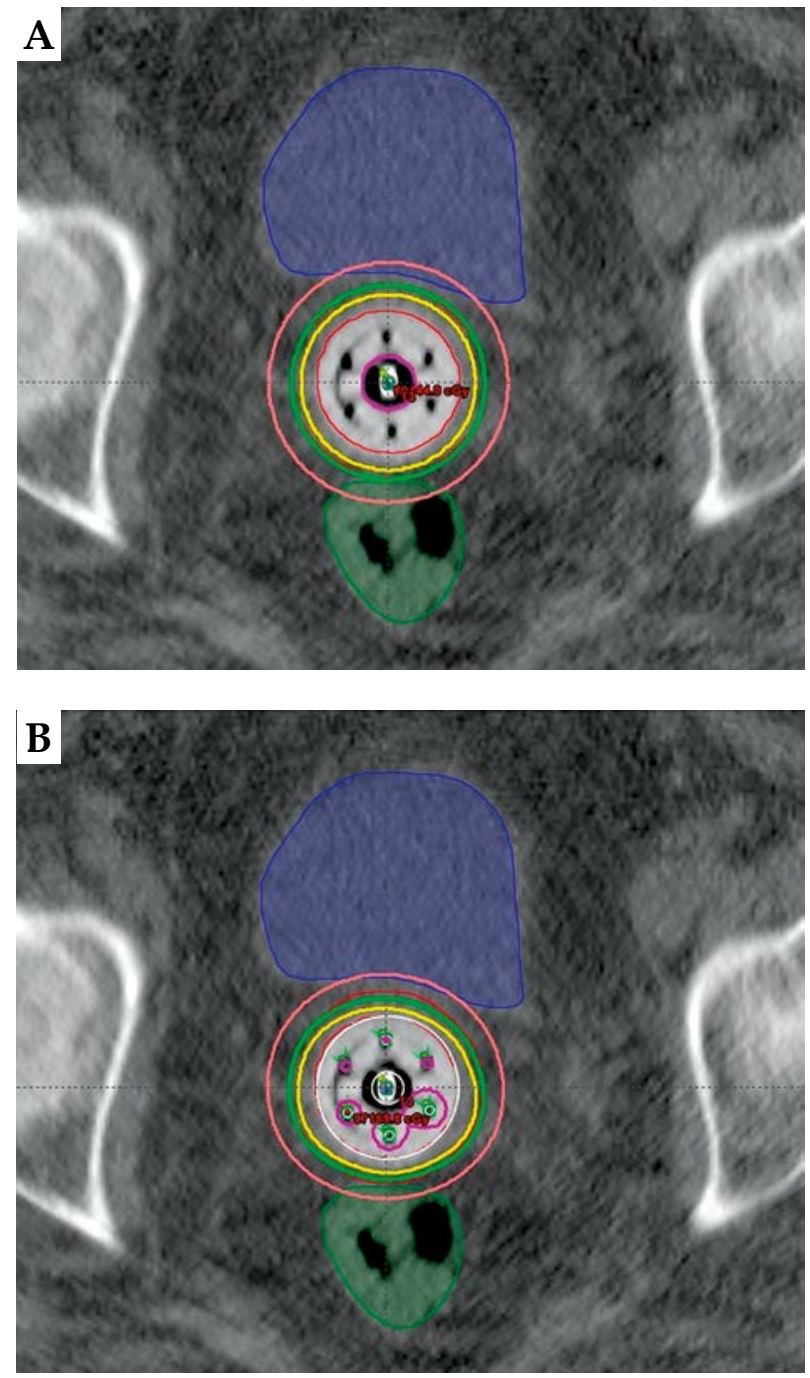

\section{Results}

Data of 12 patients who underwent CT-guided vaginal cuff HDR brachytherapy with multichannel applicators and inverse treatment planning optimization were retrospectively reviewed. Due to relatively large size of available vaginal multichannel cylinder diameter $(3$ and $3.5 \mathrm{~cm})$, not all patients could tolerate the insertion for each fraction, and their treatment was completed using a single channel applicator with smaller diameter. Two patients started the treatment with single channel applicator and, due to unfavourable plan dosimetry of single channel, had subsequent fractions with multichannel applicator, attempting for a better plan optimization. Treatment characteristics are presented in Table 1.

A total of 22 paired HDR brachytherapy inverse plans were performed and analyzed. Dose distributions and DVHs were generated for the CTV and OARs for both the central channel and multichannel approaches for all
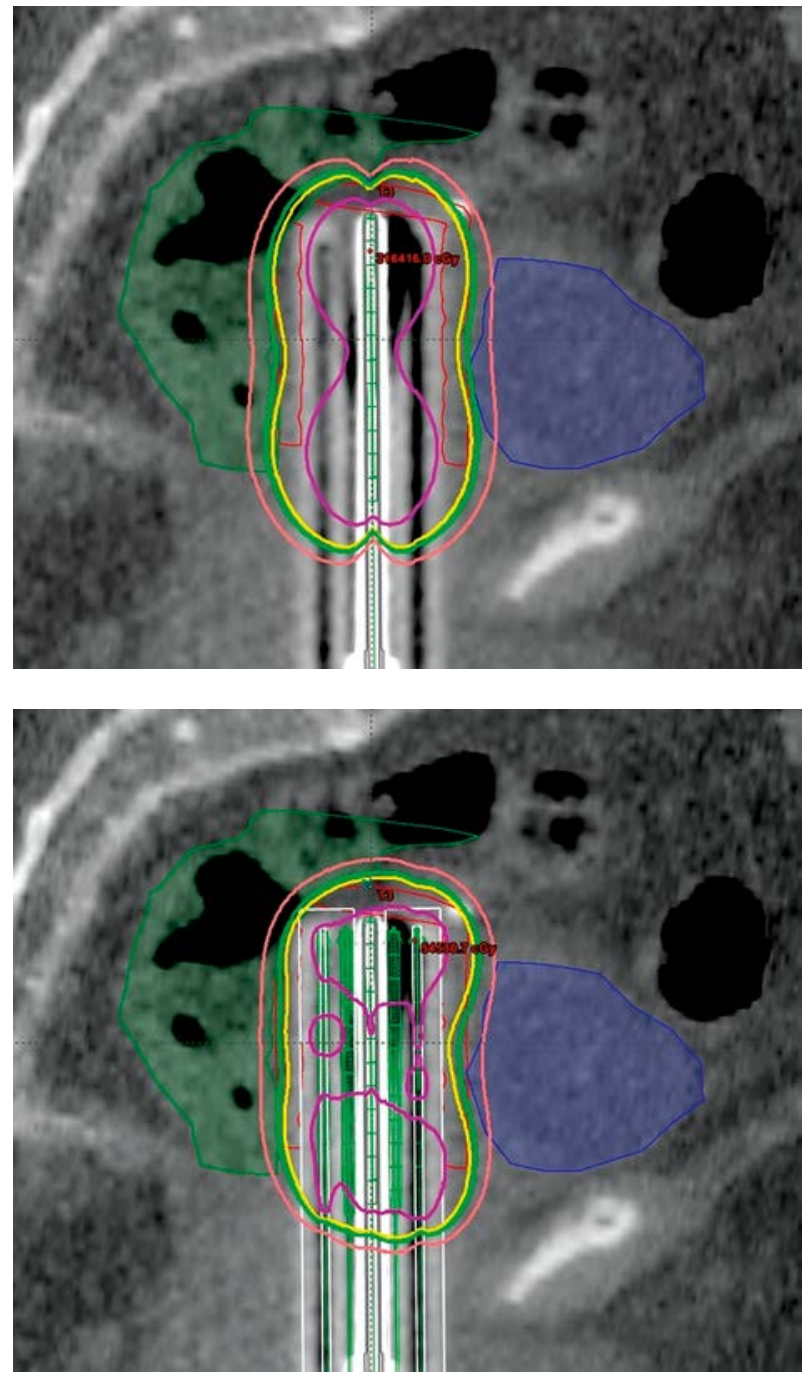

Fig. 1. Dose distribution comparison between single channel (A) and multichannel (B), in axial and sagittal views. Isodose line of $7 \mathrm{~Gy}$ (prescribed dose) is displayed in yellow, $6.3 \mathrm{~Gy}$ ( $90 \%$ of prescribed dose) in green, $4.9 \mathrm{~Gy}$ (70\% of prescribed dose) in pink and $14 \mathrm{~Gy}(200 \%$ of prescribed dose) in magenta. The capability of multichannel to correct for anisotropy at vaginal apex is clearly illustrated 


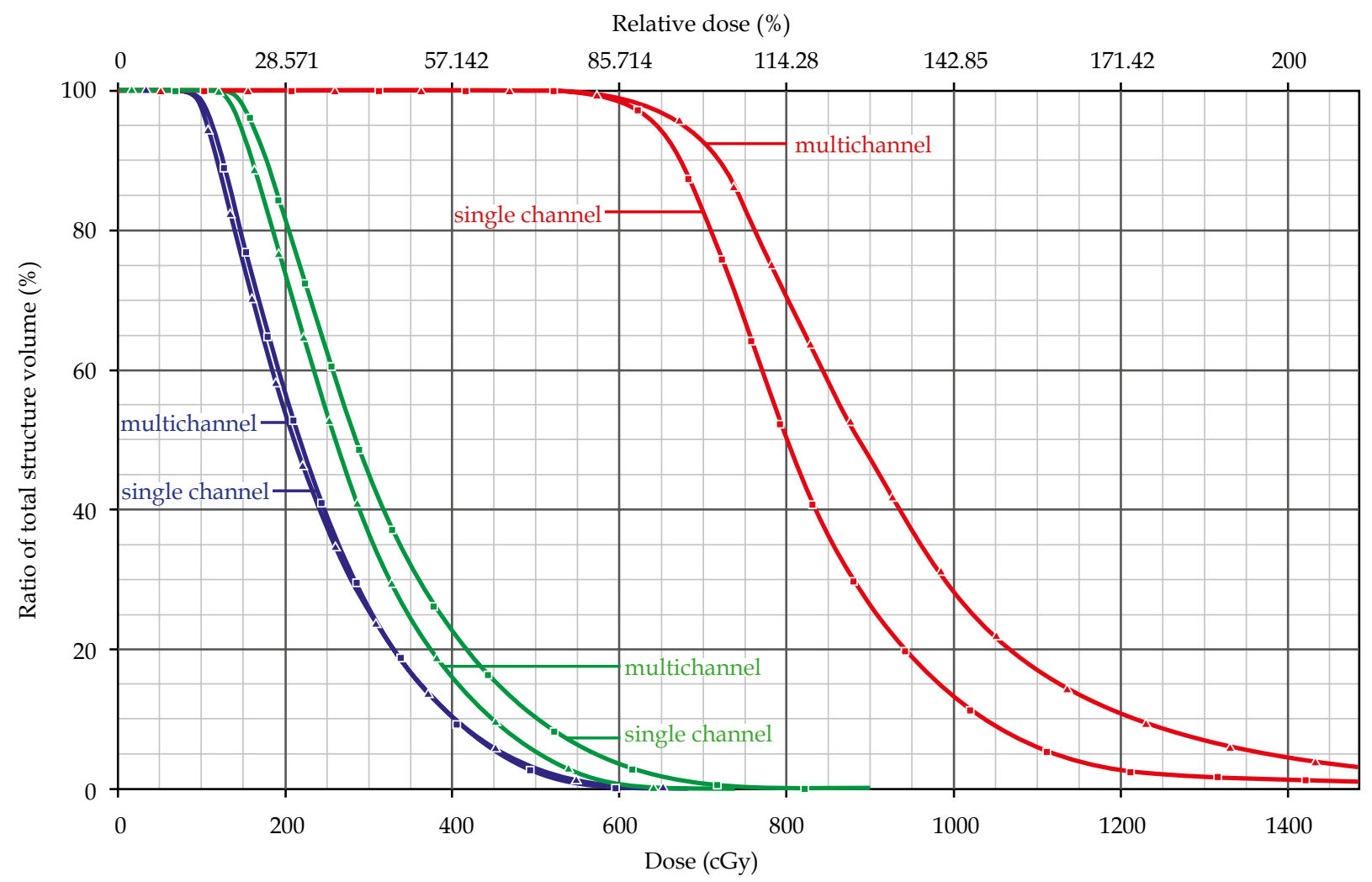

Fig. 2. Dose-volume histogram comparison between single channel (squares) and multichannel (triangles) approaches. Clinical target volume is displayed in red, rectum in green and bladder in blue

patients. Figures 1 and 2 show a comparative example of single channel and multichannel approaches, for a representative patient. The comparison is performed with respect to the dose distribution (Fig. 1) and DVH (Fig. 2).

Out of 22 brachytherapy inverse plans, none met all dose constraints for single channel, while $6(27 \%)$ fulfilled all dose constraints for multichannel. Clinical target volume dose coverage was achieved in $2(9 \%)$ single channel and $10(45 \%)$ multichannel plans. Rectum dose limit was attained in 1 (4\%) single channel and 10 (45\%) multichannel plans, and bladder in $17(77 \%)$ single channel and 21 (95\%) multichannel plans.

Rectum and bladder doses are reduced by the multichannel approach. However, limited improvement in the CTV dose coverage was achieved, but DVH analysis showed significant difference in $\mathrm{D}_{95 \%}$ of CTV between single and multichannel approaches $(p=0.007)$.

For the OARs, the use of multichannel applicator demonstrated a noticeable dose reduction, when compared to single channel, but statistically significant for rectum only $(p=0.0001)$. For $\mathrm{D}_{2 \mathrm{cc}}$ of rectum, an average fractional dose of $6.1 \pm 0.7 \mathrm{~Gy}(95 \% \mathrm{CI}$ : 5.8-6.4 Gy) resulted for single channel vs. $5.1 \pm 0.6$ Gy (95\% CI: 4.8-5.3 Gy) for multichannel. For $\mathrm{D}_{2 \mathrm{cc}}$ of bladder, an average fractional dose of $5 \pm 0.9$ Gy (95\% CI: 4.6-5.4 Gy) occurred for single channel vs. $4.9 \pm 0.8$ Gy (95\% CI: 4.5-5.2 Gy) for multichannel.

An increase of dose at $5 \mathrm{~mm}$ from the applicator tip of up to $41.3 \%$, as well as an increase of the vaginal mucosa dose of up to $34.3 \%$, was also noted in the multichannel setting. The vaginal dose at $5 \mathrm{~mm}$ from the applicator tip was significantly increased $(p=0.001)$, from a fractional average of $7.8 \pm 3.3 \mathrm{~Gy}$ for the single channel to about $9 \pm$ $2.6 \mathrm{~Gy}$ for multichannel approach. This demonstrates the capability of multichannel applicator to compensate for anisotropy effect.

Table 2 summarizes the dosimetric comparison between single and multichannel inverse plans. The dosimetric benefit of fractional re-planning was also investigated, using inverse planning optimization and multichannel applicator, for 7 patients (who had more than one multichannel insertion) and 10 paired brachytherapy plans. The DVH analysis showed large, but not statistically significant differences between first fraction plan and fractional re-planning, for all dosimetric parameters analysed, and are revealed in Table 3 and Figure 3. Patient with fraction 4 presented outstanding inter-fraction variation and was excluded from statistical analysis, because the large dose differences and small sample size would have been affected the statistical results. While the $\mathrm{D}_{2 \mathrm{cc}}$ of rectum varied with up to $23.4 \%$, the bladder appeared to be more stable to inter-fraction variations, with differences of $D_{2 c c}$ up to $11.3 \%$. The differences were due to large inter-fraction variations for rectum and bladder positioning, as well as marked inconsistencies in OARs filling, as shown in Figure 4.

\section{Discussion}

Brachytherapy has been a standard component of endometrial carcinoma therapy for over 100 years. As 
Table 2. Dosimetric comparison between single and multichannel approaches, presented as mean and standard deviation for fractional prescribed dose of $7 \mathrm{~Gy}$

\begin{tabular}{|c|c|c|c|c|}
\hline & & Single channel (mean \pm SD) & Multi-channel (mean \pm SD) & $p$ \\
\hline \multirow[t]{5}{*}{ CTV } & $\mathrm{D}_{100 \%}(\%)$ & $73.7 \pm 7$ & $74.8 \pm 6.8$ & 0.15 \\
\hline & $\mathrm{D}_{95 \%}(\%)$ & $94.7 \pm 6$ & $95.6 \pm 6.7$ & 0.007 \\
\hline & $D_{2 c c}(G y)$ & $12.3 \pm 2.2$ & $14.1 \pm 1.9$ & 0.0002 \\
\hline & DNR & $0.14 \pm 0.08$ & $0.26 \pm 0.05$ & 0.0001 \\
\hline & Ol & $0.04 \pm 0.03$ & $0.07 \pm 0.03$ & 0.0001 \\
\hline \multirow[t]{3}{*}{ Rectum } & $D_{1 c c}(G y)$ & $6.5 \pm 0.7$ & $5.5 \pm 0.6$ & 0.0001 \\
\hline & $\mathrm{D}_{2 c c}(G y)$ & $6.1 \pm 0.7$ & $5.1 \pm 0.6$ & 0.0001 \\
\hline & $V_{70 \%}(\mathrm{cc})$ & $12.2 \pm 8$ & $6.3 \pm 3.5$ & 0.0001 \\
\hline \multirow[t]{3}{*}{ Bladder } & $\mathrm{D}_{1 \mathrm{dc}}(\mathrm{Gy})$ & $5.4 \pm 0.9$ & $5.2 \pm 0.8$ & 0.081 \\
\hline & $D_{2 c c}(G y)$ & $5 \pm 0.9$ & $4.9 \pm 0.8$ & 0.053 \\
\hline & $V_{80 \%}(\mathrm{cc})$ & $1.2 \pm 1.5$ & $1 \pm 1.2$ & 0.77 \\
\hline
\end{tabular}

CTV - clinical target volume; $D_{100 \%}$ - the dose covering 100\% of volume; $D_{95 \%}$ - the dose covering $95 \%$ of volume; DNR - dose nonuniformity index DNR $=V_{150 \%} /$ $V_{100 \%} ; \mathrm{Ol}$-overdose index $\mathrm{OI}=V_{200 \%} / V_{100 \%} ; D_{1 c c}$ - the dose received by the most exposed volume of $1 \mathrm{~cm}^{3} ; D_{2 c c}$ - the dose received by the most exposed volume of $2 \mathrm{~cm}^{3} ; V_{70 \%}$ the volume receiving $70 \%$ of prescription dose; $V_{80 \%}$ - the volume receiving $80 \%$ of prescription dose

Table 3. Dose percentage differences between first fraction plan and fractional re-planning, presented as mean and standard deviation for fractional prescribed dose of $7 \mathrm{~Gy}$

\begin{tabular}{llll} 
& & $\begin{array}{c}\text { Dose difference }(\%) \\
\text { (mean } \pm \text { SD) }\end{array}$ & $p$ \\
\hline CTV & $D_{100 \%}(\%)$ & $-12.1 \pm 26.9$ & 0.12 \\
\cline { 2 - 4 } & $D_{95 \%}(\%)$ & $-9.8 \pm 18$ & 0.09 \\
\hline Rectum & $D_{1 c c}(G y)$ & $3.9 \pm 13.6$ & 0.19 \\
\cline { 2 - 4 } & $D_{2 c c}(G y)$ & $1.9 \pm 12.6$ & 0.29 \\
\hline Bladder & $D_{1 c c}(G y)$ & $1.4 \pm 6.6$ & 0.41 \\
\cline { 2 - 4 } & $D_{2 c c}(G y)$ & $0.6 \pm 6$ & 0.45
\end{tabular}

CTV - clinical target volume; $D_{100 \%}$ - the dose covering $100 \%$ of volume; $D_{95 \%}$ - the dose covering $95 \%$ of volume; $D_{1 c c}$ - the dose received by the most exposed volume of $1 \mathrm{~cm}^{3} ; D_{2 c c}$-the dose received by the most exposed volume of $2 \mathrm{~cm}^{3}$

the use of anatomy-based treatment planning for HDR brachytherapy becomes more widely used, systematic methods of dose optimization are important for quality assurance, reproducibility, and respect of the clinical issues. Although the complications rate for vaginal brachytherapy is considerably diminished than for pelvis EBRT, published reports support a need for dose optimization in vaginal brachytherapy [7-10].

Adapting a HDR vaginal brachytherapy plan to any patient specific anatomy and disease presentation using single channel cylindrical applicators is limited, as recognized by the latest ABS guidelines for adjuvant vaginal cuff brachytherapy after hysterectomy, which recommends custom applicators [3]. If a brachytherapy plan with a single channel applicator delivers unacceptable doses to the rectum and bladder, a multichannel applicator can be considered, as improves the plan dosimetry and compensates for the anisotropy at the vaginal apex [3].

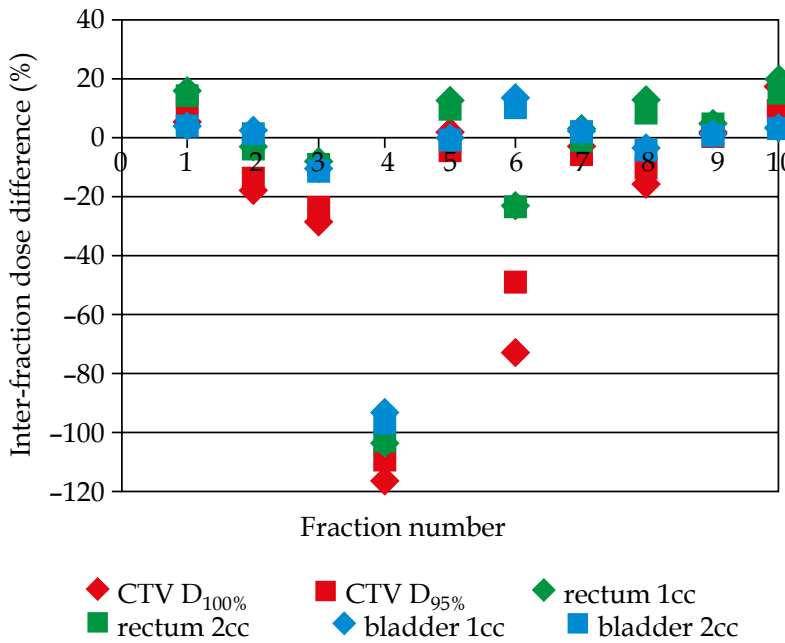

Fig. 3. Dose percentage differences between first fraction plan and fractional re-planning

Among the patients included in this study, 2 started the brachytherapy with single channel applicator and, due to unfavourable dosimetry, decision was made to complete their treatment with multichannel applicator, resulting in a better plan optimization.

The present study evaluated the magnitude of dose sparing to OARs that can be accomplished using a multichannel applicator and 3D CT-based inverse planning. Our results showed a reduction of the rectum and bladder doses, without compromising the CTV coverage. In order to maintain a uniform methodology and unbiased comparison between single and multichannel, we applied the same constraints and priorities to all treatment plans, for the purpose of this dosimetric study. However, in clinical practice, these constraints can be adapted to individual clinical circumstances. 

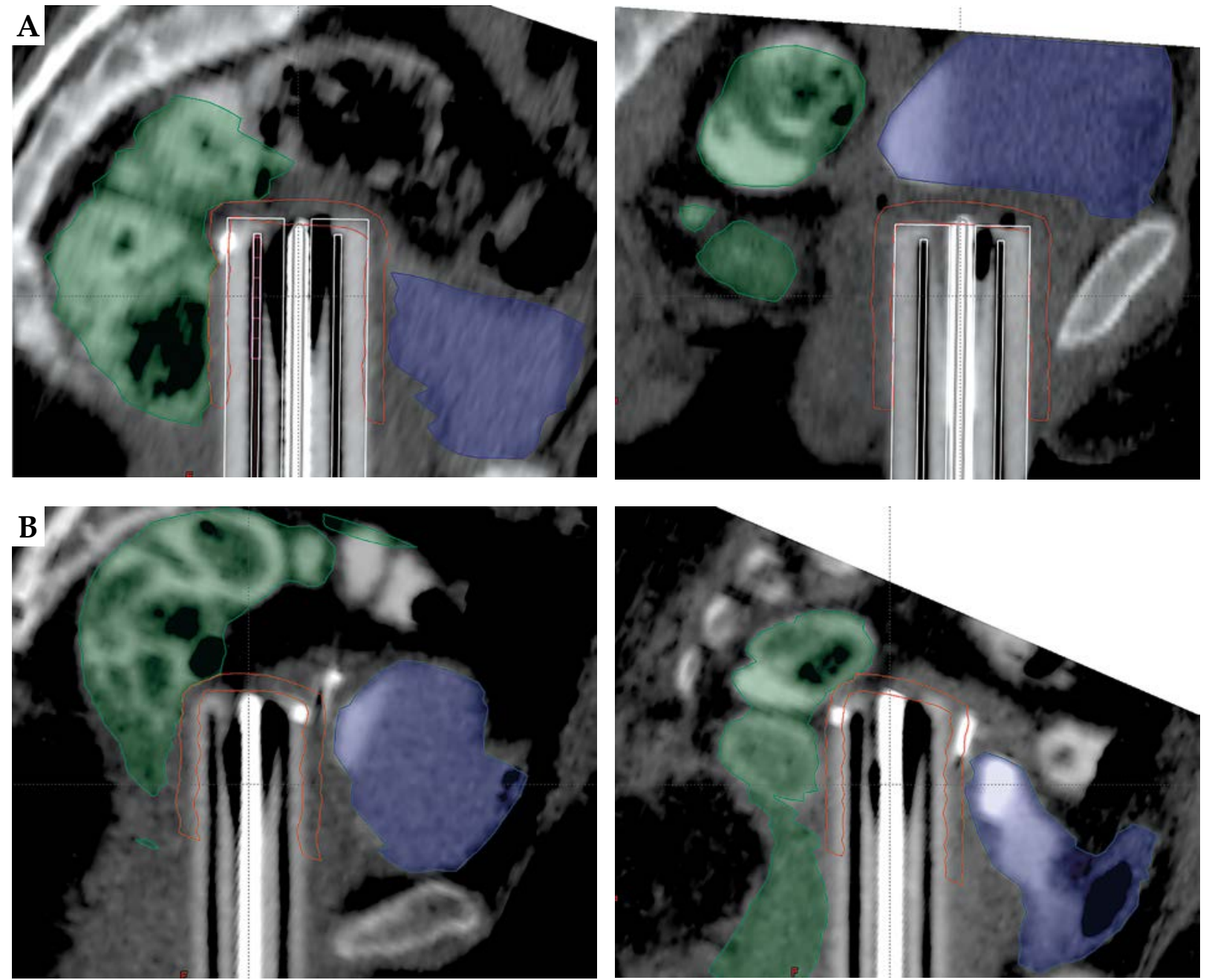

Fig. 4. The inter-fraction variation in rectum and bladder positioning (A) and filling (B), in sagittal view, for two representative patients. Figure 4A presents the patient with fraction 4; outstanding inter-fraction variations in OARs positioning is observed

With single channel, the inverse planning calculation could produce unacceptable high doses at the applicator tip and the normal tissue above, such as small bowel. The multichannel approach minimizes the effect of anisotropy and significantly improves CTV dose coverage at $5 \mathrm{~mm}$ from applicator tip by up to $40 \%(p=0.001)$. Our data revealed also a significant increase of dose to the vaginal mucosa if using a multichannel applicator of about $15 \%(p=0.0002)$, as estimated by $\mathrm{D}_{2 c c}$ of CTV. Because the source channels of the multichannel applicator are placed close to the vaginal mucosa, the dose gradient in the radial direction is steep, when compared to a single channel applicator, raising therefore the caution that the mucosal dose is acceptably increased, as recommended by ABS guidelines [3].

Although defined in interstitial brachytherapy, some plan quality indices such as DNR and OI have been used by several studies reporting intracavitary treatments, in order to assess the high dose within CTV [11-13]. Gloi et al., analysing the potential of an inflatable multichannel vaginal applicator to optimize the dose distribution in 5 patients with endometrial carcinoma, found the values of
DNR in the range of $0.1-0.3$, and OI of about 0.1 , comparable to our data: $\mathrm{DNR}=0.26 \pm 0.05$ and $\mathrm{OI}=0.07 \pm 0.03$ [13]. This value of DNR will result in a homogeneity index (DHI $=1-\mathrm{DNR}$ ) of about 0.74 , a bit higher that the value of 0.60.7 stated by ABS guidelines for interstitial brachytherapy for vaginal cancer [11]. The result is not surprising: unlike an interstitial implant, where the catheters are in direct contact with vaginal mucosa, the multichannel applicator is able to provide a slightly better dose homogeneity. Nevertheless, DNR and OI values for multichannel are two times higher than for single channel applicator $(p=0.0001)$.

To date, there are no clear guidelines regarding the acceptable high doses in intracavitary brachytherapy, and inhomogeneous dose distributions are inherently generated. Rather than controlling the high dose inside CTV by dose constraints, that might affect its coverage during inverse planning optimization process, we decided on a multicriterial approach (dose and time constraints), using the active dwell times as hard constraints forced to not exceed $300 \mathrm{~s}$ for single channel and $100 \mathrm{~s}$ for multichannel applicator, and thus eliminating possible hot spots [14]. 
As shown by other authors, the multichannel vaginal cylinders may provide more conformal dosimetry, by decreasing the dose to the rectum and bladder without a reduction in the target dose, but with the liability of a larger mucosal dose [3,15-22]. Using the flexibility of selecting source dwell positions and adjusting dwell times would result in a better shaping of individual dose distributions, compared to a single channel cylinder. An important advantage would be achieved in the specific case of treating vaginal cancer or vaginal recurrences, when the vaginal mucosa will be only partially defined as CTV $[16,20]$. Tanderup et al. have previously shown the dosimetric advantage of a 13-channels vaginal cylinder in comparison to a single channel one, in a dosimetric study using point dose optimization [21]. They reported a reduction of the hot spot doses to rectum and bladder of $17 \%$ and $16 \%$, respectively, while the vaginal mucosal dose remained the same, and recommended the use of CT-based 3D dose planning to fully exploit the capabilities of a multichannel vaginal cylinder.

The latest ABS report recognizes that, for fixed geometry applicators and assuming that the geometry of implant remains the same for each insertion, although individualized treatment planning for every fraction most accurately documents the actual total dose delivered to the patient, this practice is time consuming, involves technical costs, and may not improve patient outcomes [3]. In the particular case of vaginal brachytherapy alone, the need for routine calculation of the rectal and bladder doses has been questioned; the relatively low dose to organs at risk and the overall low morbidity of brachytherapy should be balanced against the possible risks of repeated Foley catheter insertion [3,23,24].

Several dosimetric studies, describing attempts of anatomically optimized vaginal cuff HDR brachytherapy, also investigated the benefit of fractional re-planning, but with contradicting results for both single and multichannel approaches [16-20,25-31].

Yaparpalvi et al. analyzed the inter-fraction variations of single channel applicator insertion, as well as the fluctuations in bladder and rectal volumes, which have led to variations of bladder and rectal doses; they concluded that the dose to OARs should be assessed on individual fraction basis [27]. Consequently, each fraction of vaginal cuff brachytherapy should be image-based, in order to achieve an accurate and complete dosimetric assessment of the treatment. However, other investigators stated that the small inter-fraction variation in doses to the bladder and rectum do not support treatment planning and reporting doses to the OARs beyond the first fraction $[23,32,33]$. Small concluded that the need for individualized fraction optimization for single channel vaginal cylinders is yet to be determined [26].

The OARs dose can vary considerably from fraction to fraction during the course of vaginal cuff HDR brachytherapy. A number of patient-based and technique-based factors, as: changes in bladder and rectum filling, inconsistencies in patient orientation, and differences in cylinder position within the vagina may contribute to this variation $[28,34]$. Contouring of critical organs on CT images and 3D dosimetric analysis provide a reli- able method to elucidate the nature of these daily geometric variations, despite a large inter-observer variations that has been reported $[28,35,36]$. Siddiqui et al. showed that over a series of patients, such variations result in an increased rectum volume receiving a percentage of the prescribed dose, but over the course of multiple fractions for an individual patient, this effect is dosimetrically averaged out [28].

For multichannel approach, the available data are also inconsistent. While Symon et al., analysing 44 paired brachytherapy plans, showed that individual fraction optimization is important, in order to minimize doses to critical structures, Zhou et al. concluded that there is no advantage of re-planning for each fraction, analysing the ICRU 38 points dose for rectum and bladder, in a recent dosimetric study over 9 paired brachytherapy plans and employing manual optimization $[17,25]$.

Our data demonstrate a large variation in the rectum and bladder doses, if fractional re-planning is not performed. Image analysis confirmed these findings, showing marked inter-fraction variation in OARs filling and positioning relative to the multichannel applicator. Unlike the single channel applicator, the steep dose gradient in the radial direction exhibited by a multichannel applicator seems to noticeably affect the dose to the nearby OARs, if inter-fraction variations occur. We noticed a reduction of mean values of doses to CTV, concomitant with an increase of rectum and bladder dose in the absence of fractional re-planning. These findings reveal that inter-fraction variations could be detrimental for both the local control and OARs toxicity, and emphasize the importance of image guidance and fractional re-planning, if inverse planning optimization is performed. Nevertheless, the bladder seems to be more stable to inter-fraction variations: the differences of $D_{2 c c}$ being about half of the values for rectum. A possible explanation of this result could be the filling protocol. All patients were requested to empty the rectum and bladder just prior applicator insertion, but voluntarily evacuation of bladder is usually easier than of rectum. The current literature is still lacking studies investigating the effect of inter-fraction variation on 3D inverse planning optimization for a multichannel applicator; therefore a comparison of our results, showing the importance of fractional re-planning, is not currently possible.

The outcome of HDR vaginal cuff brachytherapy relies on choosing the applicator size that would maintain a permanent contact with the vaginal mucosa and most comfortably fit the patient. However, the commercially available multichannel applicators have the minimum diameter of $3 \mathrm{~cm}$, this making them unsuitable for patients with narrow vagina or post-radiotherapy vaginal stenosis. In our clinical practice of HDR brachytherapy, the use of multichannel applicators is often challenging, despite of all our efforts (lubrication of the applicator, patient education, pain medication) to make the insertion and treatment tolerable. Out of 12 patients selected for this study, only $3(25 \%)$ were able to tolerate the insertion for all 3 fractions of brachytherapy.

Pelvic and vaginal radiotherapy may damage the vagina and produce stenosis, shortening and loss of elastici- 
ty [37-40]. Although scarcely reported, vaginal stenosis is a common side effect of radiotherapy, occurring in 38\% of patients, most often during the first year after treatment, and patients older than 50 years presents the highest risk [37]. Research has shown that maximum dose to the vaginal vault correlates with stenosis grade, and adjuvant chemotherapy before vaginal cuff brachytherapy is associated with subsequent stenosis [39]. In an effort to prevent vaginal stenosis, the use of vaginal dilators has been advocated [40].

An obvious limitation of the present study is the small number of patients data considered for statistical analysis. Such a small sample size could not be valid if generalized at patient population level, and future studies with large sample sizes are recommended. 3D CT-based vaginal HDR brachytherapy using a multichannel vaginal applicator and inverse planning optimization provides dosimetric advantages over single channel cylinder, by reducing the dose to organs at risk without compromising the coverage of target volume. However, this improvement comes at the expense of an increased vaginal mucosa dose.

For vaginal HDR inverse planning brachytherapy with multichannel applicator, our data show that large inter-fraction dose variations can occur, due to marked differences in rectum and bladder volumes and positions, therefore we recommend individual fraction optimization.

\section{Disclosure}

Authors report no conflict of interest.

\section{References}

1. Horowitz NS, Peters WA 3rd, Smith MR et al. Adjuvant high dose rate vaginal brachytherapy as treatment of stage I and II endometrial carcinoma. Obstet Gynecol 2002; 99: 235-240.

2. Pearcey RG, Petereit DG. Post-operative high dose rate brachytherapy in patients with low to intermediate risk endometrial cancer. Radiother Oncol 2000; 56: 17-22.

3. Small W Jr, Beriwal S, Demanes DJ et al. American Brachytherapy Society consensus guidelines for adjuvant vaginal cuff brachytherapy after hysterectomy. Brachytherapy 2012; 11: 58-67.

4. Nag S, Erickson B, Parikh S et al. The American Brachytherapy Society recommendations for high-dose-rate brachytherapy for carcinomas of the endometrium. Int J Radiat Oncol Biol Phys 2000; 48: 779-790.

5. Li Z, Liu C, Palta JR. Optimized dose distribution of a high dose rate vaginal cylinder. Int J Radiat Oncol Biol Phys 1998; 41: 239-244.

6. Rivard MJ, Coursey BM, DeWerd LA et al. Update of AAPM Task Group No. 43 report. A revised AAPM protocol for brachytherapy dose calculations. Med Phys 2004; 31: 663-674.

7. Dimopoulos JC, Schmid MP, Fidarova E et al. Treatment of locally advanced vaginal cancer with radiochemotherapy and magnetic resonance image-guided adaptive brachytherapy: dose-volume parameters and first clinical results. Int J Radiat Oncol Biol Phys 2012; 82: 1880-1888.

8. Nout RA, Smit VT, Putter H et al. Vaginal brachytherapy versus pelvic external beam radiotherapy for patients with endometrial cancer of high-intermediate risk (PORTEC-2): an open-label, non-inferiority, randomised trial. Lancet 2010; 375: 816-823.

9. Onsrud M, Strickert T, Marthinsen AB. Late reactions after postoperative high-dose-rate intravaginal brachytherapy for endometrial cancer: a comparison of standardized and individualized target volumes. Int J Radiat Oncol Biol Phys 2001; 49: 749-755.

10. Libby B, Sen N. Retrospective analysis of toxicity from vaginal cuff brachytherapy. American Brachytherapy Society Annual Meeting, Atlanta, GA, 2010.

11. Beriwal S, Demanes DJ, Erickson B et al. American Brachytherapy Society consensus guidelines for interstitial brachytherapy for vaginal cancer. Brachytherapy 2012; 11: 68-75.

12. Shwetha B, Ravikumar M, Katke A et al. Dosimetric comparison of various optimization techniques for high dose rate brachytherapy of interstitial cervix implants. J Appl Clin Med Phys 2010; 11: 3227.

13. Gloi AM. First clinical implementation of the Capri applicator. J Appl Clin Med Phys 2014; 15: 386-394.

14. De Boeck L, Beliën J, Egyed W. Dose optimization in highdose-rate brachytherapy: A literature review of quantitative models from 1990-2010. ORHC 2014; 3: 80-90.

15. Demanes DJ, Rege S, Rodriquez RR et al. The use and advantages of a multichannel vaginal cylinder in high-dose-rate brachytherapy. Int J Radiat Oncol Biol Phys 1999; 44: 211-219.

16. Iftimia I, Cirino ET, Mower HW, McKee AB. Treatment planning methodology for the Miami multichannel applicator following the American Brachytherapy Society recently published guidelines: the Lahey Clinic experience. J Appl Clin Med Phys 2013; 14: 214-227.

17. Zhou J, Prisciandaro J, Lee $C$ et al. Single or multi-channel vaginal cuff high-dose-rate brachytherapy: Is replanning necessary prior to each fraction? Pract Radiat Oncol 2014; 4: 20-26.

18. Peng J, Sinha R, Patel R et al. A comparison of 3D dose planning regimens for vaginal cuff brachytherapy using a flexible inflatable multichannel gynecologic applicator. Scientific Poster PD66, ABS 2011 Annual Meeting.

19. Kuo HC, Mehta KJ, Yaparpalvi R et al. Feasibility study and optimum loading pattern of a multi-ring inflatable intravaginal applicator. J Contemp Brachytherapy 2013; 5: 93-100.

20. Lapuz C, Dempsey C, Capp A, O’Brien PC. Dosimetric comparison of optimization methods for multichannel intracavitary brachytherapy for superficial vaginal tumors. Brachytherapy 2013; 12: 637-644.

21. Tanderup K, Lindegaard JC. Multi-channel intracavitary vaginal brachytherapy using three-dimensional optimization of source geometry. Radiother Oncol 2004; 70: 81-85.

22. Kim H, Rajagopalan MS, Houser C, Beriwal S. Dosimetric comparison of multi-channel vaginal cylinder high-dose-rate brachytherapy to one single channel vaginal cylinder for patients with vaginal cancer. Brachytherapy 2013; 12: S59.

23. Holloway CL, Macklin EA, Cormack RA, Viswanathan AN. Should the organs at risk be contoured in vaginal cuff brachytherapy? Brachytherapy 2011; 10: 313-317.

24. Zhu L, Craciunescu O, Cai J et al. Dosimetric impact of CTs with each fraction on organs at risk for HDR vaginal cylinder brachytherapy. Radiother Oncol 2012; 103: S110.

25. Symon Z, Menhel J, Alezra D, Pfeffer MR. Individual fraction optimization vs. first fraction optimization for multichannel applicator vaginal cuff high-dose-rate brachytherapy. Brachytherapy 2006; 5: 211-215.

26. Small W Jr. To plan or not to plan? That is the question. Brachytherapy 2006; 5: 216-217.

27. Yaparpalvi R, Shah S, Sharma R et al. Variance in bladder and rectal doses in the course of fractionated cylinder highdose-rate brachytherapy. Brachytherapy 2009; 8: 145-148.

28. Siddiqui MU, Bellon MR, Fraser $C$ et al. The nature and dosimetric effect of daily variations in vaginal cuff high-doserate brachytherapy: a prospective study. Brachytherapy 2010; 9: S89. 
29. Supe SS, Bijina TK, Varatharaj C et al. Optimized dose distribution of Gammamed plus vaginal cylinders. Med Dosim 2009; 34: 20-25.

30. Bahadur YA, Hassouna AH, Constantinescu CT et al. Threedimension anatomy-based planning optimization for high dose rate vaginal vault brachytherapy. Saudi Med J 2012; 33: 640-647.

31. Longo JM, Chen S, Thompson R et al. Measuring the interfraction variation in dose to the rectum and the bladder using CT-based treatment planning for postoperative high dose rate vaginal brachytherapy in women with endometrial cancer. Int J Radiat Oncol Biol Phys 2011; 81: S478.

32. Nixon E, Modrick JM, Jacobson GM. Retrospective evaluation of single treatment plan per course vs. plan per fraction for high-dose-rate vaginal cylinders. Brachytherapy 2006; 5: 102.

33. Nunnery EW, Ali AN, Corso C et al. Fractional re-planning with three-dimensional CT for single channel vaginal cylinder high-dose-rate brachytherapy: imaging technology overkill? Int J Radiat Oncol Biol Phys 2011; 81: S474.

34. Bellon M, Fraser C, Sintay B et al. Interfraction geometric and dosimetric variations in vaginal cuff high dose-rate brachytherapy using CT based planning: a prospective study. Int J Radiat Oncol Biol Phys 2009; 75: S381-S382.

35. Hung J, Shen S, De Los Santos JF et al. Image-based 3D treatment planning for postoperative vaginal cylinder brachytherapy: Dose-volume histograms of the bladder, rectum, and bowel. Brachytherapy 2008; 7: 148.

36. Craciunescu OI, Pepek JM, Steffey BA et al. Relevance of repeating the planning CT for HDR vaginal cylinder treatments: a comparison of intra-patient variability and inter user contouring variability. Int J Radiat Oncol Biol Phys 2009; 75: S379.

37. Brand AH, Bull CA, Cakir B. Vaginal stenosis in patients treated with radiotherapy for carcinoma of the cervix. Int J Gynecol Cancer 2006; 16: 288-293.

38. Wolf JK. Prevention and treatment of vaginal stenosis resulting from pelvic radiation therapy. Commun Oncol 2006; 3: 665671.

39. Vakilian S, Al-Suhaibani A, Bahoric B. What causes vaginal stenosis in patients undergoing adjuvant intracavitary brachytherapy for endometrial cancer? Int J Radiat Oncol Biol Phys 2011; 81: S475.

40. Johnson N, Miles TP, Cornes P. Dilating the vagina to prevent damage from radiotherapy: systematic review of the literature. BJOG 2010; 117: 522-531. 